\title{
Subwavelength Resolution in a Two-Dimensional Photonic-Crystal-Based Superlens
}

\author{
E. Cubukcu, K. Aydin, and E. Ozbay* \\ Department of Physics, Bilkent University, Bilkent, 06533 Ankara, Turkey \\ S. Foteinopolou ${ }^{1}$ and C. M. Soukoulis ${ }^{1,2}$ \\ ${ }^{1}$ Ames Laboratory-USDOE and Department of Physics and Astronomy, IowaState University, Ames, Iowa 50011 \\ ${ }^{2}$ Research Center of Crete, FORTH, Heraklion, Crete, Greece
}

(Received 10 June 2003; published 11 November 2003)

\begin{abstract}
We experimentally and theoretically demonstrate single-beam negative refraction and superlensing in the valence band of a two-dimensional photonic crystal operating in the microwave regime. By measuring the refracted electromagnetic waves from a slab shaped photonic crystal, we find a refractive index of -1.94 that is very close to the theoretical value of -2.06 . A scanning transmission measurement technique is used to measure the spatial power distribution of the focused electromagnetic waves that radiate from a point source. The full width at half maximum of the focused beam is measured to be $0.21 \lambda$, which is in good agreement with the finite difference time domain method simulations. We also report a subwavelength resolution for the image of two incoherent point sources, which are separated by a distance of $\lambda / 3$.
\end{abstract}

Recently left-handed materials (LHM) have attracted a great deal of attention since these materials exhibit a negative effective index, which is due to the simultaneous negative permeability and permittivity [1-9]. Pendry [10] proposed that a negative effective index in left-handed materials can be used for constructing a perfect lens, which is not limited by diffraction. Negative refraction is also achievable in a dielectric photonic crystal (PC) that has a periodically modulated positive permittivity and a permeability of unity [11-13]. Negative refraction and large beam steering, called the "superprism phenomenon," at the interface of a three-dimensional PC has been experimentally observed [14]. Notomi extensively studied light propagation and the refraction phenomenon in strongly modulated two-dimensional (2D) PCs [15]. Luo et al. have studied negative refraction [16] and subwavelength imaging in photonic crystals [17], where they have shown that the transmission of evanescent waves through a slab of a photonic crystal can be used to explain the focusing of light with subwavelength resolution.

In this Letter, we experimentally investigate the negative refraction and subwavelength focusing of electromagnetic waves in a 2D PC. Our structure consists of a square array of dielectric rods in air, having a dielectric constant $\varepsilon=9.61$, diameter $2 r=3.15 \mathrm{~mm}$, and length $l=15 \mathrm{~cm}$. The center to center separation between the rods is $a=4.79 \mathrm{~mm}$. The transverse magnetic (TM) polarized valence band of this structure in the first Brillouin zone, calculated by plane wave expansion method, is shown in Fig. 1(a). To study the refraction effects, the contours of equal frequency in $\mathbf{k}$ space, which are called equal frequency surfaces (EFSs), are numerically determined. Following the analysis of Ref. [16], we have determined the negative refraction range for our structure. The frequency range that gives negative refraction extends from 13.10 to $15.44 \mathrm{GHz}$. To obtain negative refraction one needs to have EFSs for the PC that are both convex and larger than the EFSs for air, which are circles with radii proportional to the frequency. The EFSs for air and $\mathrm{PC}$ at $13.698 \mathrm{GHz}$ are shown in Fig. 1(b). Note
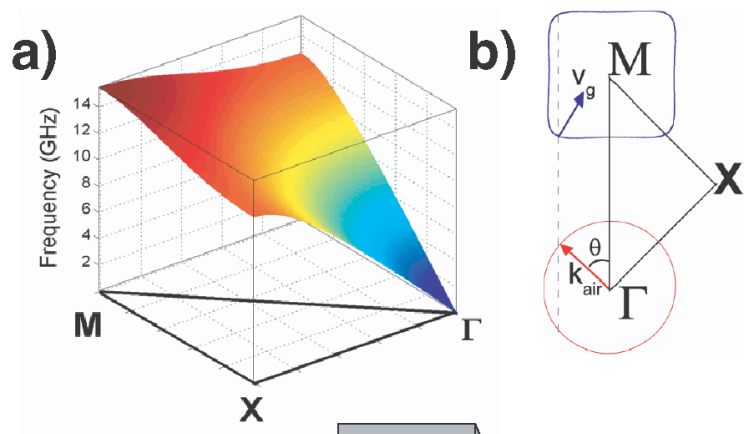

c)

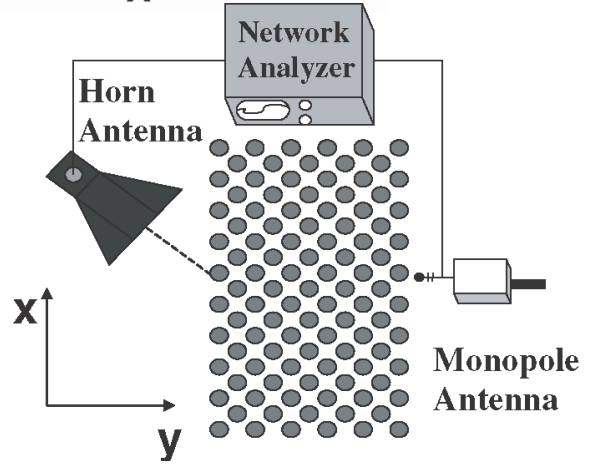

FIG. 1 (color online). (a) Band diagram for our structure for TM polarization. (b) Equal frequency contours of air and PC at 13.698 GHz. Here $\theta$ denotes the incidence angle from air to PC. (c) Experimental setup. For the refraction measurements a horn antenna is used as the transmitter, and a monopole antenna is used for focusing measurements. For both cases a monopole antenna is used as a receiver. 
that the conservation of the surface-parallel wave vector gives the direction of the refracted waves inside the PC [Fig. 1(b)].

Transmission measurements are performed for experimentally verifying the predicted negative refraction behavior in our structure. The experimental setup consists of an HP 8510C network analyzer, a microwave horn antenna as the transmitter, and a monopole antenna as the receiver [Fig. 1(c)]. The PC used in the refraction measurements has 17 layers in the propagation direction and 21 layers in the lateral direction. The interfaces are along the $\Gamma M$ direction. In all of our measurements and calculations the electric field is kept parallel to the rods. The horn antenna is oriented such that the incident waves make an angle of $\theta_{i}$ with the normal of the $\Gamma M$ interface. The operating frequency is selected as $13.698 \mathrm{GHz}$. As described later in this Letter, at this frequency our structure exhibits the maximum angular range of negative refraction. First the PC is removed and the spatial power distribution along the air-PC interface is measured by scanning the receiver antenna, which corresponds to the incident power distribution. Then the PC is placed and the spatial power distribution along the second (PC-air) interface is measured. In our experiments we can measure only the power at a certain point, which corresponds to the time averaged intensity at that point. In order to make a realistic simulation of this structure, we calculate the spatial time averaged intensity distribution along the first interface without the PC and the spatial time averaged intensity distribution along the second interface in the vicinity of the PC by using the finite difference time domain (FDTD) method. In the simulations the incident Gaussian beam width is selected to be $6 \mathrm{~cm}$, which is equal to the width of the horn antenna used in the experiment. The center of the outgoing Gaussian beam is shifted towards the left-hand side of the center of the incident Gaussian beam, which clearly corresponds to negative refraction [14,18]. Since $\lambda>2 \sqrt{2} a$, high-order Bragg reflections in our structure will not occur. Our FDTD simulations show apparent single-beam propagation in our crystal. Assuming that most of the propagating power is coupled to the zero-order diffracted wave, we can apply Snell's law in the following way:

$$
n\left(f, \mathbf{k}_{i}\right) \sin \theta_{r}=n_{\text {air }} \sin \theta_{i},
$$

where $\theta_{i}$ is the angle of incidence and $\theta_{r}$ is the angle of refraction inside the photonic crystal. $n\left(f, \mathbf{k}_{i}\right)$ is the refractive index along the propagation direction $\mathbf{k}_{i}$. The negative index of refraction determined from the experiment is -1.94 which is very close to the theoretical value of -2.06 calculated by the FDTD method for $\theta_{i}=45^{\circ}$. The advantage of refraction in the valence band of the PCs is that we do not suffer from Bragg reflections that take place inside the photonic crystal and we have a welldefined single-beam propagation that is negatively re- fracted rather than multiple Bragg waves propagating in different directions in the PC [11]. Another advantage of operating in the valence band is that we have $63 \%$ transmission at this frequency [12]. This is almost 3 orders of magnitude larger than the typical transmission in a lefthanded material [4-6].

In the aforementioned frequency range the EFSs are square shaped around the $M$ point in the Brillouin zone [Fig. 1(b)]. This results in anisotropy for $n\left(f, \mathbf{k}_{i}\right)$, where one expects to have different values for the refractive index along different propagation directions. To demonstrate this anisotropy, the refractive index of the PC for certain angles of incidence at $f=13.698 \mathrm{GHz}$ is measured. Figure 2 shows the angles of refraction for the experimental measurements and the FDTD simulations, where we observe a negative refraction behavior for the incidence angles $>20^{\circ}$. In this angular range $\mathbf{v}_{g} \cdot \mathbf{k}_{i \|}<0$ (while $\mathbf{v}_{g} \cdot \mathbf{k}_{i}>0$ ), where $\mathbf{v}_{g}$ is the group velocity inside the PC that is given as $\nabla_{k} \omega(k)$ and $\mathbf{k}_{i \|}$ is the component of wave vector, incident from air to the $\mathrm{PC}$, which is parallel to the interface. At $13.698 \mathrm{GHz}$, we achieve the maximum angular range of negative refraction for our structure. This frequency is the optimum value, because at this frequency the EFS for air has almost the same diameter as the EFS for the PC [Fig. 1(b)].

Since we know the optimum frequency for a broad angle negative refraction, we can use our crystal to test the superlensing effect that was predicted for negative refractive materials $[10,16,17,19]$. For this purpose, a PC having 15 layers in the propagation direction and 21 layers in the lateral direction is used. In our FDTD simulations, the surface of the PC is excited with a continuous wave point source that is placed at $0.7 \mathrm{~mm}$ away from the air-PC interface. An image of the source is

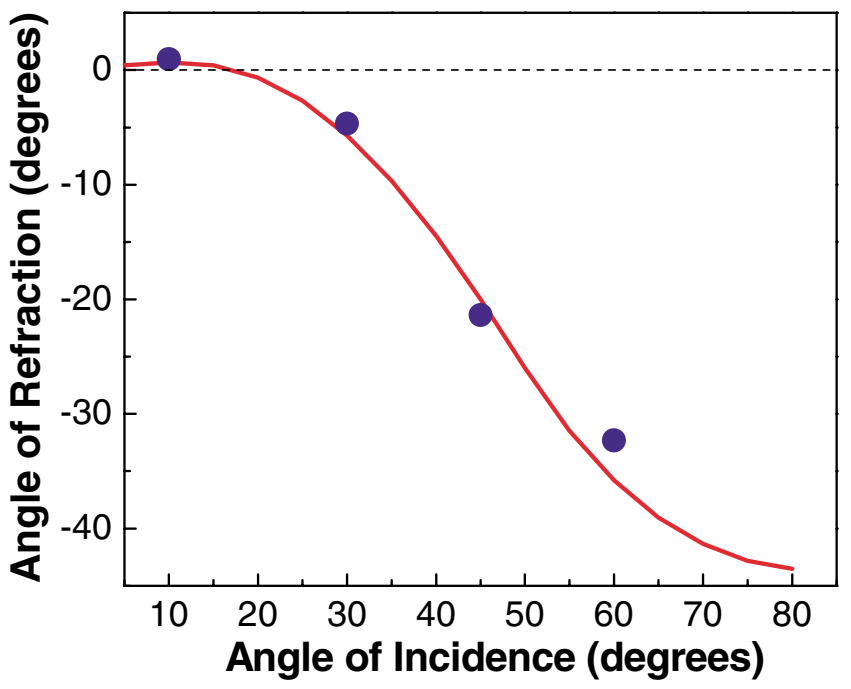

FIG. 2 (color online). Comparison of measured ( $)$ and calculated (solid line) angles of refraction versus angles of incidence at $13.698 \mathrm{GHz}$. 
formed at $0.7 \mathrm{~mm}$ away from the PC-air interface. To show the focusing on the image plane in the vicinity of the $\mathrm{PC}$, the time averaged intensity distributions along the image plane with and without the $\mathrm{PC}$ are calculated (Fig. 3). In the experiment, a monopole antenna is used as the point source [Fig. 1(c)]. The power distribution at the image plane is measured by scanning the transmission along the image plane. The measured distribution is also shown in Fig. 3. The full width half maximum (FWHM) of the measured focused beam is found to be $0.21 \lambda$, which is in good agreement with the calculated FWHM. The calculated FWHM of the beam at this plane without the PC is found to be $5.94 \lambda$. So, our structure exhibits $25 \times$ focusing at this plane with respect to free space. However, in our structure the superlensing effect can be observed only for a small separation between the point source and the PC. As the point source moves away from our finitesized crystal, only a smaller portion of the electromagnetic waves become incident on the crystal within the negative refraction incidence angle range $\left(>20^{\circ}\right)$. So, as the separation between the source and the crystal increases, the superlensing effect gets weaker and it cannot be observed for large separations. The observed subwavelength imaging can be explained by the recent theoretical work of Luo et al. [17]. As described by this reference, the subwavelength imaging is possible due to the amplification of evanescent waves through the photonic crystal. They have also found out that the periodicity of the photonic crystal imposes an upper cutoff to the transverse wave vector that can be amplified, which brings an ultimate limit to the superlens resolution. Within this description, we can also claim that there is an upper limit on the location of the source from the PC. As the evanescent waves have to reach the surface of the

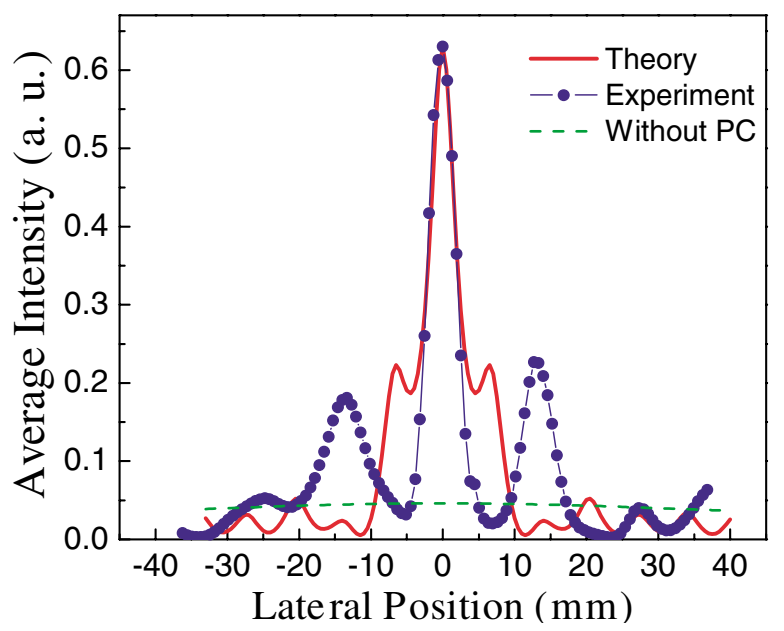

FIG. 3 (color online). Measured power distribution ( ) and calculated average intensity (solid line) at the image plane. Full width at half maximum of the measured image is $0.21 \lambda$. Spatial power distribution without PC is also shown (dashed line).
PC (before they decay out), the source has to be close enough to the PC. In that sense, the theoretically predicted and the experimentally observed subwavelength resolution in PCs will be limited to the cases where the source is very close to the PC.

A subwavelength resolution using negative refractive materials has been theoretically suggested by other researchers $[10,17,19]$. As our structure exhibits negative refraction properties, we check the possibility of observing this phenomena in our structure. For this purpose, the same structure $(15 \times 21)$ is used. First, we place two coherent point sources $0.7 \mathrm{~mm}$ away from the PC, which are separated by a distance $\lambda / 3$ or $6.78 \mathrm{~mm}$ and both having the frequency $13.698 \mathrm{GHz}$. Figure 4(a) shows the calculated power distribution at $0.7 \mathrm{~mm}$ away from the second interface with and without the PC. In the measurements the input power is split into two identical monopole antennas by a $3 \mathrm{~dB}$ splitter. The measured power distribution at $0.7 \mathrm{~mm}$ away from the second interface is also plotted in Fig. 4(a). The peaks corresponding to each point source are clearly resolved in both measurement and simulation. Since the point sources in the present experiments are obtained by the splitting of the same signal, they remain coherent with respect to each other. Thus the obtained resolution may well be due to the interference effects. So, we repeat the experiments and simulations with two incoherent point sources $0.7 \mathrm{~mm}$ away from the PC, which are separated by a distance of $6.78 \mathrm{~mm}$ and having frequencies of 13.698 and $13.608 \mathrm{GHz}$, respectively. Figure 4(b) shows the calculated power distribution at $0.7 \mathrm{~mm}$ away from the second interface of the PC. In the measurements two independent signal generators are used for driving the monopole antennas, and a power meter is used for measuring the

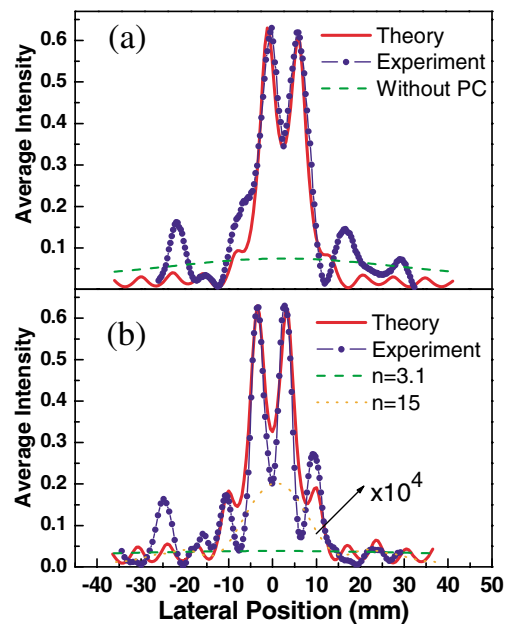

FIG. 4 (color online). Measured power distribution $(\mathbf{)}$ ) and calculated average intensity (solid line) at $0.7 \mathrm{~mm}$ away from the second interface (a) for two coherent sources and (b) for two incoherent sources. Calculated average intensities at these points with dielectric slabs are also shown (dashed lines). 
power distribution. The measured power distribution at $0.7 \mathrm{~mm}$ away from the second interface is also plotted in Fig. 4(b). The peaks corresponding to each point source are clearly resolved in both measurement and simulation. To our knowledge, this is the first experimental observation of subwavelength resolution of two incoherent sources in negative refractive materials. One can argue that the observed enhanced resolution can be due just to the large index of the refractive index as in the case of oil (or solid) immersion microscopy. In such a case, the higher wave vectors (which are evanescent in the air) can reach the crystal and then transmit through the crystal (which has a large refractive index) and can form a near-field image with subwavelength resolution. In order to check this possibility, we repeat our incoherent source simulations using a dielectric slab with a high refractive index. Figure 4(b) shows the simulations made for $n=3.1$ (refractive index of alumina) and $n=15$. As can be seen from this figure, subwavelength resolution is not achieved even with a slab made of a large index material. Besides, the large reflection resulting from the air-slab interface significantly reduces the transmitted power when compared to $63 \%$ transmission obtained from the PC at this operating frequency. So, even if the observed subwavelength resolution in our PC is a near-field effect, this cannot be achieved by ubiquitous materials

The negative refraction effect reported in this Letter depends only on the refractive index of the dielectric material and the geometrical parameters used in 2D PCs. So, this effect can also be observed at optical wavelengths where it is possible to obtain similar refractive indices using transparent semiconductors. This is in contrast to the previously reported metal-based LHMs. In such structures, increased absorption in metals prohibits the scaling of these structures to the optical wavelengths. In terms of fabrication, a slab shaped lens structure is easier to fabricate than a conventional curved shaped lens structure. So, our slab shaped lens structure can also be used for nanophotonics and nano-optics applications.

In summary, we report the experimental and theoretical demonstration of a single-beam negative refraction and a superlensing effect in $2 \mathrm{D}$ photonic crystals. Negative refraction is observed for the incidence angles of $>20^{\circ}$. This broad angle range is used to demonstrate the superlensing effect, where the electromagnetic waves emitted from a point source are focused on the other side of the PC. We also use the PC to achieve subwavelength resolution for the image of two incoherent point sources that are separated by a distance of $\lambda / 3$.

This work is supported by the European Union Project No. EU-DALHM, DARPA Contract No. MDA 972-01-20016, and NATO Grant No. SfP971970. Ames Laboratory is operated for the U.S. Department of Energy by Iowa State University under Contract No. W-7405-Eng-82.

*Corresponding author.

Email address: ozbay@fen.bilkent.edu.tr

[1] V. G. Veselago, Usp. Fiz. Nauk 92, 517 (1964) [Sov. Phys. Usp. 10, 509 (1968)].

[2] J. B. Pendry, A. J. Holden, D. J. Robbins, and W. J. Stewart, IEEE Trans. Microwave Theory Tech. 47, 2075 (1999).

[3] J. B. Pendry, A. J. Holden, W. J. Stewart, and I. Youngs, Phys. Rev. Lett. 76, 4773 (1996).

[4] D. R. Smith, W. J. Padilla, D. C. View, S. C. Nemat-Nasser, and S. Schultz, Phys. Rev. Lett. 84, 4184 (2000).

[5] R. A. Shelby, D. R. Smith, and S. Schultz, Science 292, 77 (2001).

[6] M. Bayindir, K. Aydin, E. Ozbay, P. Markos, and C. M. Soukoulis, Appl. Phys. Lett. 81, 120 (2002).

[7] D. R. Smith, S. Schultz, P. Markos, and C. M. Soukoulis, Phys. Rev. B 65, 195104 (2002).

[8] P. Markos and C. M. Soukoulis, Phys. Rev. B 65, 033401 (2002).

[9] P. Markos and C. M. Soukoulis, Phys. Rev. E 65, 036622 (2002).

[10] J. B. Pendry, Phys. Rev. Lett. 85, 3966 (2000).

[11] B. Gralak, S. Enoch, and G. Tayeb, J. Opt. Soc. Am. A 17, 1012 (2000).

[12] E. Cubukcu, K. Aydin, E. Ozbay, S. Foteinopoulou, and C. M. Soukoulis, Nature (London) 423, 604 (2003).

[13] S. Foteinopoulou, E. N. Economou, and C. M. Soukoulis, Phys. Rev. Lett. 90, 107402 (2003).

[14] H. Kosaka, T. Kawashima, A. Tomita, M. Notomi, T. Tamamura, T. Sato, and S. Kawakami, Phys. Rev. B 58, R10 096 (1998).

[15] M. Notomi, Phys. Rev. B 62, 10696 (2000).

[16] C. Luo, S. G. Johnson, J. D. Joannopoulos, and J. B. Pendry, Phys. Rev. B 65, 201104 (2002).

[17] C. Luo, S. G. Johnson, J. D. Joannopoulos, and J. B. Pendry, Phys. Rev. B 68, 045115 (2003).

[18] J. A. Kong, B. I. Wu, and Y. Zhang, Appl. Phys. Lett. 80, 2084 (2002).

[19] D. R. Smith, D. Schurig, M. Rosenbluth, S. Schultz, S. A. Ramakrishna, and J. B. Pendry, Appl. Phys. Lett. 82, 1506 (2003). 